\title{
VARICOSE ULCERS
}

\author{
By H. W. EDWARDS
}

(Continued from January, 195.5)

\section{THE APPLICATION OF THE THERAPY TO VARICOSE ULCERS.}

Care must be taken that the path of radiation from radiation head to the skin, is completely occupied by a contact substance. If the surface is injured, it is necessary to direct the waves obliquely to the surface, irradiation in a water bath should then be carried out. The irradiated parts of the skin should be rendered wettable, as far as possible, by soaping or brushing them over with alcohol. The relevant parts of the body should then be dipped in a vessel of water, (not too cold)? Air bubbles should be removed with a soft brush. These also form frequently during irradiation, and are perceptible often as a mild burning surface pain. The radiation head is moved in an up-and-down or circular manner, under the water, about $1-2 \mathrm{~cm}$. away from the skin.

Ulcers are always treated in a water bath, never by massage, as' the danger of introducing infection exists. Movement of the head should take place around the ulcer, and extend into healthy tissue, far away from the edge of the ulcer. The intensity of irradiation is $0.5-2.0$ watts per sq. $\mathrm{cm}$. and the more dystrophic the surroundings, the less the intensity. The treatment is given for 5 minutes daily, for not more than 10-20 times.

\section{THE BISGAARD MÉTHOD.}

Bisgaard in 1939 described the ambulatory treatment of ulcers, in which the patient has plenty of exercise and leads an active life. Healing by these methods takes time, but is more satisfactory.

This treatment is designed at improving the circulation to the leg, and increasing the mobility of the joints. The deep and superficial areas of infiltration are reduced, and oedema controlled.

A combination of massage and movements, with a specialised type of dressing and bandage, correctly applied, fulfil these designs.

The treatment should be given twice a day, and patients should be able to apply their own bandages. Plenty of exercise is advocated, and the importance of walking five miles a day should be stressed.

(a) Massage:

First Stage.-The leg is put up in elevation, with a pillow under the buttocks, to avoid kinking of the femoral vein. This aids the emptying of the venous system. It is advisable to leave the leg in this position for 10-15 minutes before commencing massage.

The area around the ulcer is massaged with deep finger and thumb kneadings, gradually encroaching on the edge of the ulcer. The kneadings should get deeper and deeper during the course of treatment, and should continue for $10-20$ minutes, depending on size of ulcer. Zinc Oxide and $2 \%$ menthol ointment is usually used, being mildly antiseptic and stimulating to the circulation.

Second Stage.-The leg is examined for areas of infiltration. These areas are gradually encroached upon with kneadings, which become deeper and deeper. 5-10 minutes treatment to each area.

Third Stage.-Using effleurage and kneading, the whole leg is massaged, avoiding the ulcerated areas and varicose veins. A 5 minute treatment is given.
Massage should be given at least twice a day, once by the physiotherapist and once by the patient at home. This should be done, preferably when going to bed and if possible on getting up in the morning. Patients' co-operation is essential.

(b) Dressings.

The open area is cleaned with a special solution. This is a mixture of aluminium acetate and boracic acid, $1 \%$ Aluminium Acetate, $0.3 \%$ Boracic Acid. Gauze of 16-20 thicknesses, cut to the size of the ulcer, is soaked in this solution and applied to the ulcer area with firm pressure.

The area directly around the ulcer is protected with.a layer of Zinc Oxide and Menthol Ointment, to prevent the surrounding tissues from becoming soggy. Over the piece of gauze, is placed a thin piece of non-absorbent cotton wool, followed by a piece of oil silk, applied for even pressure and to prevent moisture from leaking out.

A cotton bandage holds the dressing in place. Strips of wadding are cut, a 2 -in. strip to fit around the foot, a 5 -in. strip round the malleoli and a 2 -in. strip round the calf, just below the knee. It depends on the size of the Patient's shoes whether the necessary cotton wool can be applied. A special elastic webbing bandage is used, 3 in. wide and approximately $11 \mathrm{ft}$. long, depending on the size of the leg. This is known as the Meisen bandage, and gives much greater support than any other type of bandage. it is made of rubber and cotton threads, both lengthwise and only cotton crosswise. Hence the bandages are nonelastic in a transverse direction. Natural rubber should be used, as synthetic rubber may cause eczema. Both edges of the bandage should be hemmed.

(c) Method of Application of Bandage:

First turn: applied behind the metatarso-phalangeal joint of the big toe, so that the bandage lifts the internal edge of the foot, and thereby counteracts flat feet. A turn is taken round the foot to secure bandage in position.

Second turn: goes round the foot and lies vertically to the second turn, on the inner side and is known as the "stirrup." Each time the patient takes a step, this gives a double massage where most needed. This turn is fixed on the tubercle of the Navicular.

Fourth turn: goes round the malleoli and continues in successive turns up the leg to the popliteal space, where it is firmly secured. Each time, approximately half of the previous turn, must be covered, the proximal side of the bandage being slightly stretched, and the distal part slightly looser, to allow for the previous turn. An even pressure is thus exerted. The bandage must give firm support to the ankle, particularly to the grooves behind and below the malleoli, and edges and base of the ulcer. Movement must not be restricted, and patient must be able to wear shoes which are properly fitting. The bandage should be removed at night.

\section{Function of bandaging:}

The turn around the heel behind the malleoli, has a massaging effect as the patient walks. This is increased by the wadding. The turn under the sole of the foot, which is fixed on the navicular, supports the plantar vessels.

The bandage up the leg acts as a slight counterpressure on muscular activity, and therefore has a pumping effect on the underlying blood vessels. 
Rhythmic exercises of the toe, ankle and knee joints with the bandage applied, assists in reduction of oedema. Patients are taught to move all the joints of the lower extremity, and to walk without a limp. The pumping action exerted by the bandage helps to reduce swelling and soften induration.

The healing process can be hastened by giving ultraViolet Light to the actual ulcer. Patients who are not able to walk five miles a day, are given sinusoidal or faradism under pressure.

The patients should be instructed in home treatment, leg elevation for 10 minutes with exercises and a 10 minute massage to ulcer area. Method of bandaging is most important.

The size of the ulcer should be recorded, by tracings at regular intervals. Healing in most cases takes $3-6$ months, although the first signs should be noticeable after $2-4$ weeks.

\section{ADDITIONAL MEANS OF TREATMENT.}

\section{(a) Exercises:}

In addition to the rhythmic mobility exercises employed in the Bisgaard method, "riding a bicycle" in the air and "Buerghers" exercises are both used. To counteract venous stasis, the patient should be taught to do heel raising frequently. Good heel-toe walking is a necessity.

\section{(b) Lotions and Ointments:}

In addition to the Zinc and Menthol ointment used by Bisgaard, others used are:-

Olive Oil, Lanoline, Zinc and Pyrogallol, Cod Liver Oil, and Sulphonilamide. Injection of Tincture of Iodine is said to aid healing. The local use of antibiotics has not proved of great value.

\section{(c) Bandaging:}

Other types of bandages - crepe and elastic, are used and applied in a manner similar to Bisgaard's elastic webbing, but they do not afford the same amount of support.

McPheeters \& Anderson believe in bandaging as follows: A 4-in. bandage rolled tightly, is started at the flexion crease, just below the knee and wrapped around the leg vrey tightly twice. Wrapping is continued down the leg, overlapping about two thirds of the width of the bandage, with each turn. It is carried twice about the ankle and foot and fastened securely. Patients must walk quickly for short distances, every three hours. The bandage may be removed at night, and re-applied first thing in the morning. It is obvious, however, that this method is in no way comparable to the Bisgaard method.

\section{(d) Rubber Sponge Treatment:}

By pressure of a sponge and an elastic bandage, the reverse flow of blood in the venous system into the ulcer area, can be overcome. Excessive accumulations of fluid in the tissues about the ulcer, which causes a lowered resistance locally, can be stopped.

By means of its resilience, the rubber sponge, when bound firmly in place by the bandage, causes a constant, soft, gentle but firm massage of the area beneath it, when the patient is walking. This transmits the massaging effect to the blood in the veins, with each contraction of the calf muscles, hence the more often and the faster the patient walks, the more rapid is the healing of the ulcers.

The sponge is placed in the middle of the area, after it is covered with ointment and dressings. The ulcer and skin surface about it, are covered with gauze, to which the ointment has been applied. A good grade rubber sponge can be used, and should extend 1 in. beyond the edge of the ulcer. It is applied over several layers of fluffed gauze, to absorb the discharge. Wadding is applied over the sponge and then bound in place with cotton gauze bandage. A strong elastic bandage is then wrapped around the leg. It is applied tightly and evenly. The tighter the bandage, the more quickly the patient will be relieved of pain and the more rapid the recovery. Though walking is painful for the first hour or two, the patient must persist, and if too severe, a sedative tablet can be given. If discharge is extensive, bandage and dressing should be changed every day, otherwise every 2 nd to 4 th day.

With this treatment, the large, coarse watery granulations will be killed off and a fine, normal granulating area will develop. Secretion lessens, necrosis disappears and the general appearance of the ulcer becomes normal. The tissues surrounding the ulcer will lose their dark, congested appearance and look more healthy. The rubber sponge can be discarded, when the appearance of tissues becomes nearly normal, but the support with compression bandage must be continued until ulcer is healed and for a long time after healing.

\section{(e) Myer Fisher's Treatment:}

In 1949, Myer Fisher advocated the use of hot saline or magnesium sulphate packs in the treatment of ulcers. These were applied for one week. Infra-Red at a distance of 18-in., for a period of 15 minutes, was used until the local swelling had subsided. The ulcer and surrounding skin was then irradiated with Ultra-Violet Light by means of an Alpine Lamp or Kromayer until a good erythaema was produced. Supportive bandaging was employed when the ulcer was healing.

\section{AFTER-CARE.}

This may be described as the most important stage in the treatment of any varicose ulcer, if a permanent cure is to be obtained. For, although it may be comparatively easy to remove the underlying cause and to cure the ulcer, the patient still has a tendency to develop varicosities in other veins and form further ulcers. The-co-operation of the patient is essential, as, if the self-care is neglected, a recurrence is inevitable.

The sense of futility and helplessness of so many patients should be removed, and their interest in the treatment obtained, to avoid any morbid tolerance of their ulcers and discharges. Patients should, therefore, be informed of the pathology present, etiology, causative factors, circulatory conditions and the theory of the supportive treatment. They should be made to realise that it is not possible to restore the normal physiology to the limb, but should remember the Chinese proverb:-

"The used doorstep never rots".

It cannot be over-emphasised that the patient must be fully aware of the complications which will result if instructions are not strictly adhered to. Although cured, it is only through supervision and self-care that such complications can be avoided.

The general health of the patient must be improved and can be done best by fresh air, healthy surroundings and physical exercises. In this connection, the patient should be instructed in personal hygiene and general cleanliness. The gravitational factors in the causation of ulcers, must be impressed upon the patients, who should change their way of life and adapt their work to minimize them. Where necessary, the importance of weight reduction should be stressed. Obesity should be treated with thyroid and even in non-obese cases, a small dose of thyroid is beneficial. (Sicard).

Home-massage and exercises should be taught and practised by the patient during healing, and for some time after the ulcer has healed.

The wearing of some kind of support and participation in exercise, frequently leads to a permanent return of tone in the calf muscles, as waterlogging is prevented. The patient may ultimately be able to dispense with the bandages and stockings, but for several months after the immediate cure of the ulcer, it is most important for the leg to be fully supported. If this support is not maintained, the 
leg will become oedematous and tissues cyanotic in a matter of days.

The types of supportive bandages commonly employed are:-

\section{(a) Semiplast:}

An elastic plaster bandage similar to elastoplast, but half the width is spread with Zinc Oxide paste, and half is plain elastocrepe; only on the first turn does the medicant come into contact with the skin.

This is applied in the same manner as the Bisgaard bandage, and is worn 3-4 weeks following cure of ulcer. It reduces the leg to normal proportions and is intermediary to the permanent wearing of a crepe bandage.

(b) Viscopaste:

An Adhesive gelatin bandage (Zinc Oxide and gelatin mixed). It serves a similar purpose as the semiplast-less elasticity and becomes looser in wearing. Indicated only when swollen legs are mildly eczematous.

\section{(c) Ichthopaste:}

Same as the Viscopaste with the addition of ichthyol, which cleanses unhealthy skin surrounding the ulcer. Used in the treatment of varicose eczema.

(d) Crepe and "elastocrepe":

When legs are reduced to normal by removal of all superficial and deep oedema, they must be maintained in a healthy condition. A bandage should be worn in the daytime and applied as before. Elastocrepe is better than crepe, because it gives more support and has more elasticity, a longer life further lengthened by washing, does not pull out or fluff and is more durable. They are moderately priced.

A lighter crepe silk is available, which is less bulky, cooler and better in appearance.

\section{(e) Bisgaard Bandage:}

This is by far the most efficient means of support, and when procurable should always be recommended.

\section{(f) Elastic Stockings:}

Some patients find bandaging too much trouble or too difficult to apply and unattractive when showing through stockings. Special cotton stockings are available, having considerable elasticity and being made to measure. These last for several months and are washable.

Patients of heavier build are advised to wear the elastic bandage during the day, and the stocking only for social occasions.

If the patient perspires freely, the stocking becomes uncomfortable and rots.

The patient must avoid all trauma to the area, as the tissues are not yet able to sustain the destructive effect of injuries.

A system of following up cases after treatment must be organised. Without these measures little progress in the subject can be expected. Records of all cases, with details of exact treatment, together with all information as to drugs and supportive measures, must be meticulously registered.

A plan for photographic records of exceptional cases, taken before and after treatment, is an ideal, which would offer valuable help in furthering the progress of methods of treatment. In any case, tracings of ulcers should be taken at each attendance, and an exact note of improvement or retrogression should be made.

When an ulcer is cured, it is necessary to give the patient written instructions regarding the further care of the leg.

A suggested list of instructions which may be suitably modified for the case in question is:-

Your ulcer is now healed. It will come back again if you do not carry out these instructions. Your co-operation is essential.

\section{COMPLICATIONS AND THEIR TREATMENT.}

\section{(a) Recurrence:}

The commonest cause of recurrence is that of low intelligence, usually associated with poor and dirty home conditions. Trauma plays a large part.

An analysis showed that all cases had discarded their bandages and had discontinued massage. These cases of re-ulceration necessitate complete treatment again.

(b) Varicose Eczema, or Stasis Dermatitis:

This precedes and occurs with ulceration, and is generally found around the malleoli, associated with varicosities. Another type of dermatitis results from absorption of a medication, to which the patient has become hypersensitive. Sulfonamide preparations are common causes and some patients become hypersensitive to their own secretion and eruptions develop.

Impairment of nutrition to the skin is the chief underlying factor, producing stasis dermatitis. 1t starts off with eczematous patches, which may become vesiculated and oozing with a crust formation.

The skin is bluish-red and later hyperpigmented. Oedema may be slight or severe. Trauma, especially scratching, with secondary infection, aggravates and maintains the condition.

Treatment :

Any offending veins must be treated. The part must be cleansed with warm boric acid solution, a moist compress applied or area softened with olive or mineral oil. For acute cases with oozing, rest in bed is advisable. Support is most necessary and should be maintained for weeks, even after the skin has apparently. returned to normal. Unnas' boot may afford valuable relief.

Careful hygiene of the feet is necessary.

\section{(c) Idiosyncrasy to Zinc Oxide:}

Occasionally severe reactions occur with a severe dermatitis of the leg and foot. Calamine lotion and rest for a few days remedies this.

In rare cases patients cannot tolerate Zinc Oxide under tight pressure bandaging. Dr. Fynan's method is to prepare a weak solution of Boric acid, and soak 3-ply white surgical lint in this solution. This is bandaged on to the ulcer, extending for 1-in. beyond the ulcer.

Painting the skin of the leg and foot with collodium, prior to the application of elastoplast, is a preventative against the risk of "plaster dermatitis".

(d) Cellulitis:

This is one of the commonest complications, and is due to a streptococcus infection involving the superficial fat and fascia. It is a low grade and chronic type of infection, which at times seems to flare up and resemble an erysipelas. The area is red, sore and tender; oedema is present which is painful. In the chronic stage, the tissues are firm and hard, and bronze in colour. The superficial fat seems to burn up and the skin tightens.

Treatment.--Sulfadiazine in large doses with hot wet packs. If oedema is present a supportive bandage is required. Deep X-ray therapy is useful.

(e) Pigmentation:

The skin is in a poor condition in most cases, being dry, scaly, eczematous and oedematous. With ulceration, the red blood cells break down and their haemoglobin is freed. This leaves a permanent dark brown patch.

Pigmentation is only irritant from a cosmetic point of view.

(f) Indurated Scar:

Following the ulcer there is a scar, which is non-elastic, avascular and bound down. lt is usually tender.

Treatment.-Daily scar massage with gentle finger kneadings round the edges, and exercises to stretch the scar. 


\section{(g) Deformities of the feet:}

It is very rare to find a patient with leg ulcers having normal feet. Owing to pain and fear that movement is harmful, the patient has probably not used the foot for months. Deformities such as pes planus and hallux valgus develop. The patient thus walks in the most comfortable position, usually with a limp. The deformities must be treated by the physiotherapist. The fitting of proper shoes is essential.

\section{(h) Sudden arrest of healing:}

A large ulcer at first heals rapidly, but when about 1-in. in diameter healing sometimes ceases. The dressings should then be changed and the Ultra-Violet Light dosage increased. The Sponge Rubber method of treatment often assists at this stage.

\section{CASE HISTORIES.}

\section{A. Mrs. L. Age 72. Housewife. Admitted to hospital} $4 / 3 / 1954$.

The patient complained of severe pain in the ankles. She was shot in the leg in the Boer War, and treated in 1904. Since then she has had a stiff left ankle, and has had to walk on her toes. An ulcer developed on the outside of her left ankle, which would get better for 6-12 months, but always returned.

About two years ago, the patient knocked her right ankle, which remained painful for about a year before it ulcerated. It was not as painful as the left ankle and could be moved slightly. One year ago the ulcers were treated with Unnas' paste. This was of little avail. A month ago her ulcers became much worse, and she was hospitalised. They were septic and not responding to home therapy.

Systematic History:

All the systems were normal and healthy, but the patient suffered from nerves and headaches.

\section{Left Lower Limb:}

The leg had been swollen for the past 50 years. The pulses were present but the left ankle jerk very weak. Over the lateral malleolus, there was a large septic, sloughing ulcer, roughly 3 -in $\times 4$-in. The edges were irregular but not undermined or rolled. The base had reddish granulating areas, interspersed with yellow septic areas. There was a brown pigmentation of the skin around the ulcer, which extended halfway up the leg and on to the foot.

A similar picture was observed over the medial:malleolus, where an ulcer 4 -in $\times 2$-in. existed. The ankle was grossly swollen and stiff and could be moved neither actively nor passively. It was fixed at about $110^{\circ}$ to the foot.

Right lower limb:

The leg had been swollen for the past $1 \frac{1}{2}$ years. The pulses were present and right ankle jerk normal. There was a sloughing septic ulcer over the right lateral malleolus, and a recently healed one over the medial malleolus. The ankle was oedematous and there was a brown pigmentation of the skin. Movement was present but limited. The toe nails of both feet were thick and unhealthy looking, and tended to become detached.

\section{Treatment:}

Conservative methods were employed. The foot of the bed was raised, Eusol and dry dressings were applied. The patient was then put on to Physiotherapy treatment, UltraViolet Light and the Bisgaard Method.

The ulcers immediately improved with a vast difference in the patient's outlook. The case, however, was not suitable for skin grafting, due to the probability of recurrence.

\section{B. Dr. H. Age 54. Dental Surgeon.}

The patient had had varicose veins on both legs since 1914. These were very slight on the right leg, but prominent on the left leg. He had had no treatment.
In 1928, the veins were injected, but the operation was a failure. Ulcers developed above the left ankle, but never occurred on the right leg.

In 1932, the ulcers were healed after 3 months by elasto. plast bandaging.

In 1939, another ulcer broke out below the ankle, and was cured after 6-8 weeks by the rubber sponge pressure method. They re-occurred in 1942 and would not respond to any treatment. Bandaging and sponge rubber pressure were tried together with ointments and lotions, but there was no response.

The veins were then ligated, and the ulcer disappeared, but the operation was otherwise not successful. There was a tendency for ulcers to develop, but none actually broke out. In May 1954, there was a further recurrence. A history of trauma to the skin area was recorded earlier in the year.

This ulcer was below the medial malleolus, about $\mathrm{J} \frac{1}{2}$-in. long and 1-in. wide, with painful, tender areas round the ulcer. There were areas of induration above the malleolus, together with marked pigmentation from previous ulcers. Veins in the ankle region were prominent and hard to the touch, and obvious throughout the lower leg.

The patient had flat feet for a number of years. Treatment:

The Bisgaard method and Ultra-Violet Light treatments were used. Ultrasonics were introduced but after a few days the ulcer became soggy, and this treatment was discontinued. The ulcer began to heal, tender areas lessened and the legs felt less heavy with this supportive means of treatment.

Treatment is still continuing and ulcer improving.

C. Mrs. C. Age 58. Housewife.

The patient had varicose veins for many years, and high blood pressure for three years, for which she was treated.

In 1950 , she sustained a slight injury to the lower tibial region. She was re-injured later in the same place, where an ulcer broke out and became septic. This was present on the lower aspect of the left leg. Ulcer healed after 6-7 months, but pitting oedema and pigmentation were still present. A Crepe bandage was given to support the varicose veins, and because of her obestity.

In 1954, she was re-admitted with an ulcer on the lateral side of the lower portion of the left leg. The ulcer was about 4 -in $\times 3$-in. and the skin around the ulcer was red, but not oedematous. The pulses were present. The ulcer was seeping with an evident cellulitis.

\section{Treatment:}

The area was cleaned with Eusol, Tulle gras and gauze dressings were applied.

A Viscopaste boot was applied, and was to be kept on for four weeks. Two weeks later the patient was back, having tried to remove the boot. The old one was then removed and a new one applied. Two weeks later the patient was back again, having once again tampered with her boot. The Ulcers were much the same, and the Viscopaste boot was re-applied, this time for 8 weeks. After 8 weeks, the patient came back with the leg in a dreadful state, having interfered with it.

She was immediately hospitalised and put on to a Sulphonamide dressing, Ultra-Violet Light and Short Wave treatments were started, and patient made good progress. Bisgaard treatment was introduced, and the ulcer was healing well.

\section{CONCLUSION.}

Varicose Ulcers are the most common type of leg ulcers. They are common because of the low intelligence or indifferent attitude of some persons, as almost invariably there is a history, which indicates neglect at some stage. The predisposing pathological conditions have probably been operating for years. 
providing that specialised advice is sought early enough, Providing of ulceration and its complications, could be the outbreak of ulceration and

Certain occupations may be partly responsible for ulceration, as gravitational factors are of the utmost importance. tion, as grang venous stasis, with lowered tissue resistance, The resulting vossible loss of valve function, all lead to tissue and the poss of which the immediate cause may be trivial. hreakdown, of which the sufficient The symptoms of ation to be avoided. These include: warning for ulceration to be ances of the skin; infiltrations of obvious nutritional of fluid into the tissues; pain and variabnormal effusions of fluid if correctly and adequately applied at this stage, ulceration may be avoided.

In the text, numerous methods of treatment are discussed, In is obvious that only the modern form of ambulatory but it is should be seriously considered. It has been nroved that patients with ulcers, always show considerable provrovement once hospital treatment is given, no matter what methods are applied. Extreme care must therefore be exercised in evaluating the effect of a particular form of therapy.

Each ulcer must be treated individually, and a differential diagnosis made, to ensure that the correct treatment is given, and that the underlying cause is rectified. Although, in special cases, a particular treatment is obviously the best, for a general routine method the Bisgaard, with modifications, cannot be surpassed.

Surgery alone may cause an ulcer to heal, but can never effect a permanent cure. Medicine, in the form of ointments and lotions, has no direct value in bringing about a permanent recovery, and should only be used as an adjunct. It is thus only Physical methods which can fulfil all the aims of treatment, and ensure a permanent cure. As a general rule, conservative methods should always be preferred to more drastic procedure.

The Physiotherapist, who is in a position to integrate all the methods available, and select the correct combination for each case, plays a most important part in the rehabilitation of the patient. However, the co-operation on the part of the patient is vital, as home-treatment must be carried on after the actual hospital or clinic period.

The outlook for patients with Varicose Ulcers is a bright one to-day, if the patient is able and willing to co-operate with the Physiotherapist, who applies the most modern and effectual treatments.

\section{BIBLIOGRAPHY.}

1. The Physiotherapists' Role in the Treatment of Varicose Ulcers.-R. ROWDEN FOOTE, B.J. of P.M., March 1954.

2. Physiotherapy and the Indurated Leg.-R. RowDEN FoOTE, B.J. of P.M., August, . 1953.

3. A short account of the Treatment by Physiotherapy of Gravitational Ulcers.-AUDREY E. BARTHOLOMEW, $B$. J. of P. M., December, 1952.

4. Physiotherapy in the Treatment of Varicose conditions of the Lower Limbs.-E. M. BRAdiNG, B. J. of P. M., October, 1952

5. The Treatment of Gravitational Ulcers by Physiotherapy.-Audrey E. BARTHOLOMEW, $B$. $J$. of $P$. $M$., October, 1952.

6. Varicose Veins \& Ulcers and their Treatment by the Bisgaard Method.-S. A. S. of P., April, 1951.

7. From the use of Hyaluronidase in Chronic Ulcers.Milton ENDE, 1951

8. Division of the Popliteal Vein in the Treatment of socalled Varicose Ulceration, GUNNAR BAUER, B. J. of $P$. M., August, 1950 .

9. Ulceration of the Legs. Treatment by Bisgaard Method. -P. Bouwers, B. J. of P. M., Sept.-Oct., 1949.

10. Physical Medicine in the Treatment of Varicose Veins \& Indolent Varicose Ulcers.-MYER FISHER, B. J. of $P$. M., May-June, 1949.
11. Injection treatment of Varicose Veins and Haemorrhoids.-McPheETERS \& ANDERSON, 1946.

12. Varicose Veins, Haemorrhoids and other conditions.R. ROWDEN FOOTE, 1944.

13. The Cure of Haemorrhoids and Varicose Ulcers.Stuart McCausland, 1934.

Madam,

\section{LETTER TO THE EDITOR.}

Js there no way in which we can stimulate interest and enthusiasm among the readers of this Journal, particularly with a view to exchanging ideas? Surely, someone, somewhere has a little "wrinkle" he or she is proud of and through the medium of the Journal can give the benefit of experience to others.

So much is happening in the Physiotherapy world, but a great deal of useful knowledge is confined to "the few."

I seem to remember so many delegates at the last Conference were enthusiastic about writing letters and articles and promised to encourage others to do so. Were these just empty words to be forgotten as soon as they returned to their home ground?

We are particularly keen here to pick other people's brains and are only too glad to have our own picked. I am sure there is much valuable knowledge just waiting to be put on to paper.

Otherwise, where are those letters and articles? Are they arriving? Have you not printed them, or are we as a Society lacking in ideas and initiative? Please tell us what has happened?

Medical School,

JEAN BLAJR.

University of the Witwatersrand.

I can only conclude that Miss Blair is correct and members do lack enthusiasm and initiative, since despite many promises, no material has arrived for publication.-EDITOR.

\section{BRANCH NEWS}

\section{SOUTHERN TRANSVAAL.}

On February 8th, Miss Estelle Alberts, who has recently returned from working at Stope Mandeville Hospital, gave a most interesting lecture on the treatment of paraplegics at that Hospital. The lecture, which is reproduced in this issue, was illustrated by slides, and it was gratifying to see a large audience attend this function.

On February 22nd, Dr. H. Haden talked to the Branch about his recent experiences in America and his impressions of rehabilitation there. Among other things, he gave a talk on the Kabat method of muscle re-education, and Miss E. Botting demonstrated this method on a patient suffering from polio. This was a most stimu ating talk and has encouraged many of us to try this new conception of treatment, particularly for our polio patients.

\section{NORTHERN TRANSVAAL.}

Miss' J. Crewe-Browne and Miss J. von Schrader are welcomed as new members of the Branch.

The following Associate members are also welcomed: Miss Sieburg, Miss Wilson, Miss Slone, Miss van der Spek, Miss Grunow, Miss Morgan, Miss van Woudenberg, Miss Millar, Miss Silverman and Miss Hitchcock.

A successful symposium on polio wsa held in February, and in March Mr. van der Spuy gave a most interesting talk and showed a film on Thoracic Surgery. 